J. R. Tarchanoff: Ueb. d. willkürliche Acceleration d. Herzschläge etc. 109

\title{
Ueber die willkürliche Acceleration der Herzschläge beim Menschen.
}

\author{
Von \\ Prof. J. R. Tarchanoff, \\ in St. Petersburg. \\ Hierza 6 Holzschnitte.
}

Obwohl die Herzaction der Willenseinwirkung direct in der Regel nicht unterworfen ist, verfügen wir bereits heute iiber eine nicht geringe Zahl von Beispielen, für die intime Abhängigkeit des Herzschlags, bezüglich Frequenz wie Character, von dem psychisehen Leben des Menschen.

Entsprechend den zwei typischen regulatorischen Nervenbahnen, die mit dem Gehirn das Herz verknüpfen, den hemmenden und accelerirenden Nervenfasern, beeinflussen die verschiedenen psychischen Zustände die Herzaction bald durch Verlangsamang, bald durch Beschleunigung der Pulsationen.

Auf das enge Abhängigkeitsverhältniss der Herzaction von den psychischen Gehirnfunctionen wurde nicht ein Mal und mit besonderer Ueberzeugungskraft von Kürschner ${ }^{1}$ ), Carpente ${ }^{2}$ ), Claude-Bernard ${ }^{3}$ ) u. A. hingewiesen. Es ist leicht, durch flüchtiges Aufzählen einiger auf diese Frage bezïglichen Facta, die Existenz eines solchen Abhängigkeitsverhältnisses ausser jeden Zweifel zu setzen.

Jeder weiss es, wie dentlich das Gebiet der Geftihle und Affecte den Rhythmus des Herzschlags beeinflusst: angenehme und frendige Gefühle und Affecte acceleriren, unangenehme oder traurige deprimiren oder hemmen den Herzschlag.

1) Kürschner, Herz und Herzthätigkeit; Wagner's Handwörterbuch der Physiologie, 1844. Bd. II, p. 82-84.

2) Carpenter, Principles of the Human Physiology, 1864, p. 735.

3) Claude-Bernard, Leçons sur les propriétés des tissus vivants, $1866, p .465$.

E. Pflüger, Archiv f. Physiologie. Bd. xxxv. 
In dieser Beziehung erseheint besonders lehrreich ein Fall von Prof. Botkin, betreffend einen Patienten, der an progressiver Muskelatrophie litt und die seltene Eigenthïmlichkeit besass, nach Willkür und zur beliebigen Zeit sehr auffällig den Herzschlag hemmen und dessen regelmässigen Rhythmus stören zu können. Zum Hervorbringen dieser Erscheinung branchte derselbe seine traurige Lage sich nur vorzustellen. Frendige Gedanken riefen jedoch das Gegentheil nicht hervor ${ }^{1}$ ).

Die höchsten Grade heftiger und plötzlicher Empfindung von Freude oder Trauer sind sogar im Stande, momentanen Herzstillstand hervorzubringen. Diese Erscheinungen sind zu bekannt, um uns länger auf́zubalten.

Interessanter sind die Fälle, die in dem Rhythmus des Herzschlags auch die Abspiegelung des Denkprocesses beweisen.

Millner Fothergil12) beschrieb unlängst folgendes interessante Factum: Beim Auscultiren des Herzens mit dem Stethoscop an einem nervösen jungen Menschen bemerkte er, dass jede an denselben gerichtete und von Seiten desselben Nachdenken erfordernde Frage unverzuiglich eine Verlangs amung der Herzschläge hervorrief, wobei der Puls änsserst unregelmässig wurde; die den Denkprocess nicht in Anspruch nehmenden Fragen blieben ohne jeden Effect auf den Herzschlag.

Es ist ausserdem bekannt, dass anhaltend auf's Herz concentrirte A ufmerksamkeit die Funktion dieses Organs verändern könne. So begann Fran $k^{\circ}$ ) beim Lesen über die Herzkrankheiten auf die Schläge des eigenen Herzens seine Aufmerksamkeit zu lenken; es trat bei ihm in Folge dessen ein äusserst unregelmässiger, intermittirender Puls auf. Erst eine Zerstreunngsreise befreite ihn von den aequirirten Anfallen.

Damit barmonirt völlig auch ein anderes Factum von Morgagni, der einen Bologneser Professor dadurch vom intermittirenden Pulse befreite, dass er ihm rieth, niemals selbst seinen Puls zu zählen ${ }^{4}$ ).

1) Klinische Wochenschrift. Herausgegeben von Botkin. 1881. No. 10 (russisch: Eschenedelnaja Klinitscheskaja Gazeta).

2) Millner Fothergill, Gaillard's Medical Journal, Febr.1880, p.161.

3) Joseph Frank, Praxae medicae universae praecepta, Lipsiae, Th. II. Bd. II. Abth. II, p. 373.

4) Wagner's Handwörterbuch d. Physiol. 1844, Bd. II, p. 82. 
Angesichts des so augenscheinlichen Einflusses der verschiedenen psychischen Momente auf den Herzschlag läge es nahe zuzulassen, dass auch der Wille, so oder anders, den Herzsehlag reguliren könnte, und es dürften, wie es scheint, zu Gunsten dieser Hypothese folgende Thatsachen angeführt werden.

Tü ke erwähnt eines Mitgliedes der Londoner königlichen Gesellschaft, der, 79 J. alt, dem Autor experimentell seine Fähigkeit, nach Wunseh um 10 oder 20 Schläge in der Min. den Herzschlag zu acceleriren 1 ) bewies; andererseits wird in der Litteratur eines Lieutenants Townsend erwähnt, der die merkwürdige Fähigkeit besass, willkürlich Herzschlag und Athmung anzuhalten und eben damit in einen todesähnlichen Zustand zu verfallen: der Körper begann sich abzukühlen und erstarrte gleichsam, die Augen wurden unbeweglich, und zuletzt schwand das Bewusstsein; binnen einigen Stunden kam er wieder zu sich. Er starb endlich nach einem ähnlichen Experiment, das er vor vielen Zuschauern ausgeführt hatte, noch am Abend desselben Tags. Die Section ergab keine Organdegenerationen, mit Ausnahme der rechten Niere' ${ }^{2}$ ).

Dieselbe Fähigkeit, das Herz anzuhalten, besitzt ein wohlbekannter amerikanischer Physiolog ${ }^{3}$ ).

In beiden Fällen wurde der Stillstand des Herzens bewirkt ohne jeden äusseren Druck auf den Vagus, $d$. h. ohne jene Manipulation, mit Hülfe deren der Prager Professor Czermak den Schlag seines Herzens hemmen und zum Stillstand bringen konnte.

Auf die angeführten Fälle einer anscheinend willkürlichen Acceleration des Herzsehlags soll jedoch vorsichtig Bezug genommen werden, seitdem besonders von Weber der Beweis geführt wurde, dass Aenderungen des Respirationsrhythmus direct die Pulsfrequenz beeinflussen: rare Respiration, die durch tiefe Inspirationen unterbrochen wird, bewirkt auffällige Verlangsamung der Herzschläge einerseits durch intrathoracische Druckveränderung,

1) Daniel Hack Tüke, Mllustrations of the Influence of the Mind upon the Body in Health and Disease, designed to elucidate the Action of the Imagination; London, 1872.

2) Symond's; Miscellanies, 1871, p. 160, und Carpenter, Human Physiology, 1855, p. 1103.

3) Millner Fothergill 1. c. p. 160. 
andererseits durch Begïnstigung der Kohlensäure-Anhäufung, wodurch die in der Medulla oblongata gelegenen hemmenden Herzcentren gereizt werden; frequentes, oberflächliches Athmen führt zum Gegentheil. Weber hat an sich selbst demonstrirt, dass durch Anbalten der Respiration in der Inspirationsstellnng und energische Contraction der Brustmuskeln bei geschlossener Stimmritze (d. h. bei Verhinderung der Exspiration) völliger, tiefe Ohnmacht bedingender Stillstand des Herzens hervorgerufen werden könne.

Ausdrüicklich so erklärt sich Weber den Mechanismus des an dem Lieutenant Townsend anscheinend willkiulich erfolgten Stillstandes des Herzens ${ }^{1}$ ).

Beim Prïfen der Web er'schen Experimente an vielen Personen unter den angedeuteten Bedingungen erhielt Donders: Schwinden des Pulses, des Herzstosses und der Herztöne ${ }^{2}$ ).

Auf Grund obiger Data müssen die Fälle der anscheinend willkürlichen Acceleration oder Verlangsamung der Herzschläge jedes Mal bezüglich des Entstehungsmodus Zweifel wecken, d. h. ob nicht welche die Herzregulation beeinflussende Nebenmomente daran participiren, wie z. B. willkurlich hervorgerufene Ideen von dem oder jenem Character, willkürliche Aenderungen des Respirationsrhythmus u. s. w. und nicht der direcs auf die Herzregulatoren wirkende Willenseinfluss.

Eine solche Analyse war, soviel uns bekannt, auf keinen der bekannten Fälle, wenn anch nur mit Hulfe der graphischen Methode, angewendet worden und deshalb besitzen dieselben gar keine Beweiskraft für unsere Frage.

Beim Darstellen eines, meiner Ansicht nach schlagenden Falles von willkürlicher Acceleration des Herzschlags, war ich natürlicher Weise bemïht, so viel wie möglich alle so oder anders verkniupfte Momente zu durchforschen behufs einer Erklärung des Mechanismus dieser Erscheinung. Mit Hülfe der gehörigen Apparate verfolgte ich graphisch die Veränderung von Frequenz und Character des Pulses, die Veränderung der Respiration and die

1) Weber, Ueber ein Verfahren, den Kreislauf des Blutes und die Funetion des Herzens willkürlich zu unterbrechen, Arch. f. Anat. und Physiol. und wissenschaftl. Medicin, von J. Müller, 1851, p. 88 u. f.

2) Donders, Weitere Beiträge zur Physiologie der Respiration und Circulation. Zeitschr. f. ration. Medic. 1854, p. $241 \mathrm{f}$. 
Umfangsschwankung der Extremitäten während der Accelerationsperiode wie anch vor und nach derselben; gleichzeitig verfolgte ich auch die Schwankungen des arteriellen Blutdrucks und die Vertheilung der Hauttemperatur. Eine solche Untersuchung gewährte interessante, rein objective, für die Analyse unseres Falles kostbare Data. Nur ein rein subjectiver Factor, der a priori an der Acceleration des Herzschlags participiren könnte, wir meinen die willkürliche Hervorrufung dieser oder jener, die Herzaction accelerirender Ideen oder Vorstellangen, entzog sich der Untersuchung. In dieser Beziehung musste ich aus Nothwendigkeit den Aensserungen des zu Untersichenden mich anvertrauen, dessen Worten zu glauben wir allen Grund hatten, denn es war ein ernstlich gebildeter Jïngling, der den Ernst der Untersuchung erkannte und für die correcte Erklärung seiner seltenen Gabe, den Herzschlag zu acceleriren, zugleich sich lebhaft interessirte.

Das uns interressirende Individuum, ein Student aus den obe. ren Classen der Medico-Chirurgischen Academie, Eugen Salomé, ist von hohem Wuchs, eher mager und etwas nervös und empfindsam. Zwischen dem zehnten und fünfzehnten Lebensjahr litt er an Herzklopfen, das anscheinend ohne jede äussere Veranlassung aufgetreten war. Im Laufe der Zeit verminderten diese Anfälle sich fast bis zum Schwinden unter dem Einfluss einer vorgenommenen Behandlung und anderer günstigen Bedingungen, und er hätte das fast vollständig vergessen, wenn nicht seine Studiengenossen auf die sonderbare Fähigkeit seines Herzens, bei relativ unbedeutender äusserer Veranlassung den Pulsrhythmus auffällig zu verändern, die Aufmerksamkeit gelenkt hätten. Auf diese äusserste Irritabilität seines Herzens die Aufmerksamkeit concentrirend, bemerkte einst Salomé zufällig, dass es genüge, die Herzschläge nur acceleriren zu wollen und in dieser Absicht die gehörige Willensanstrengung aufzuwenden, um die gewtinschte Wirkung zu erreichen.

Bei unserer ersten Begegnung gab er schlagende Beweise seines Könnens, and acelerirte den Herzschlag von 70 auf 105 , d. i. um 35 Schläge in der Minute. Dieses Experiment wiederholte er mit demselben Erfolge einige Mal, obzwar der Grad der Acceleration mit jeder Wiederholung deutlich abnahm.

Von der Zuverlässigkeit der Sache iiberzengt, benutzte ich H. Salomé's liebenswürdige Proposition and stellte eine Reibe 
von Experimenten und Beobachtungen an ihm an, um die wahre Natur der anscheinend willkürlichen Acceleration der Herzschläge zu eruiren.

Die erste Präsumption wäre, dass Salomẻ zum Zweck der Pulsacceleration irgend welche angenehme oder freudige, mit Pulsacceleration gewöhnlich sich associirende Vorstellungen hervorrufe. Dann böte die Acceleration bezïglich des Modus der Entstehung eine nabe Analogie mit den Erscheinungen der willkürlichen Erzeugung z. B. der Gänsehaut, Speichelsecretion u. s. w. In diesen Fällen werden ja die Effecte durch willkürliche Production der entsprechenden Phantasiebilder hervorgebracht, im ersten Fall der Kälte, im zweiten - des Schmeckhaften oder Sauren, und es orscheint als die unmittelbare, Gänsehaut wie Speichelsecretion bestimmende Bedingung nicht der Willensimpuls, sondern die durch denselben vermittelte Zwischenvorstellung.

Allein wir sind nicht berechtigt, in unserem Falle eine solche Analogie zuzulassen, da Salomé kategorisch behauptet, dass er zur Acceleration der Herzschläge weder Ideen noch Vorstellungen hervorrufe, sondern auf die Schläge des Herzens die Aufmerksamkeit nur leicht concentrire und zum Zweck der Acceleration des Herzschlags vollkommen bewusste Willenskraft aufwende. Diese Willenskraft gleiche in der Qualität vollkommen der bei willkürlicher Contraction beliebiger Muskelgruppen bemerkbaren Empfindung. Während der Sitzung habe er allerdings ein manchmal unbestimmtes Gefühl einer Contraction oder eines Gespanntseins der Halsmuskeln und sogar in der Herzregion, aber dies bisweilen und bei weitem nicht immer. Das Gefühl der Aufspannung ist jedoch von objectiven Zeichen der Contraction der Hals- oder Brustmuskeln nicht begleitet.

Es ist wichtig hier zu bemerken, dass nach Salomé's Worten die blosse Concentration der Aufmerksamkeit auf die Herzaction dem Zweck der Acceleration in der Regel nicht geniige, sondern dass dazu auch Anstrengung der Willensthätigkeit nothwendig sei. Es wiederholt sich hier gleichsam dieselbe Erscheinung, wie sie in der Sphäre willkürlicher Bewegungen der quergestreiften Körpermuskeln besteht.

Es ist bekannt, dass eine Anstrengung oder Contraction von Muskeln innerhalb bestimmter Grade die Herzaction gewöhnlich accelerirend beeinflussen. Es musste demnach verfolgt werden, 
ob nicht der Untersuchte, um auf diesem Nebenwege die Pulsacceleration hervorzubringen, seine Muskeln errege. Er verbrachte die Zeit, während welcher er die Herzschläge accelerirte, in verschiedenen Körperlagen, im Liegen, Sitzen, Stehen, und es gelang

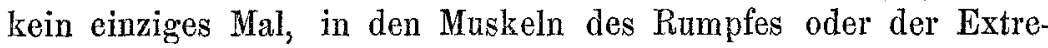
mitäten merkliche Contractionen oder gar Spannungsveränderungen zu erhaschen; in der Regel erschien das Muskelsystem im Zustand völliger Rahe, und nur merkliches Erröthen des Gesichts und eine geringe, während der Sitzung nicht immer auftretende Spannung der Halsmuskeln zeugten von irgend einer Anstrengung in der Accelerationsphase. Beiläufig sei bemerkt, dass sein Hals vollkommen normal entwickelt und keinerlei Tumoren bietet.

Bei der Beribrung der Frage über das Muskelsystem unseres Individuums erscheint es nicht ohne Interesse, dass er dasselbe in aller Vollkommenheit beherrscht; er vermag besonders solche Muskeln willkürlich zu verkürzen, die der Willenseinwirkung in der Regel entgegen sind; so bewegt er die Ohren, contrahirt den M. platysma myoides beiderseits combinirt oder jeden isolirt, flectirt willkürlich jede beliebige dritte Finger-Phalange, contrahirt die versehiedenen Muskelgruppen der Hüfte isolirt u. s. w.

Der den Willensimpulsen unterworfene Herzmuskel erscheint darnach gleichsam als ein specieller Fall dieser seiner ganz besonderen neuromuseularen Organisation.

Aus den eitirten Arbeiten von Weber, Donders n. A. ist es bekannt, dáss Character wie Rhythmus der Respiratiosbewegungen sich deutlich in der Frequenz der Herzschläge abspiegeln. Es musste demnach entschieden werden, ob in unserem Falle die Herzacceleration nicht das Resultat willkürlicher Aenderungen der Respirationsbewegungen sei. Dazu waren Experimente in folgender Form angestellt.

Auf dem berussten Papier eines gleichmässig und horizontal sich bewegenden Cylinders Balzar'scher Construction wurden gleichzeitig 4 Curven gezeichnet (Fig. 1).

Die erste derselben wurde mit dem Marey'schen Chronographen gezeichnet und jede Zacke bezeichnet eine Secunde.

Die zweite wurde von der Feder des electrischen Signales von Deprés aufgetragen and bezeichnet die Zeit des Ruhezustandes des zu Untersuchenden in Form eines einfachen weissen Striches, während ein weisser breiter Streifen die Zeit der Acce- 
lerationsperiode darstellt. Letzterer wurde dadurch erhalten, dass der zu Untersuchende gleichzeitig mit dem beginnenden Willensimpulse behufs Acceleration des Herzschlags mit dem Zeigefinger auf das Ende eines horizontalen Hebels leise drückte, wobei durch das Emporsteigen dessen anderen Endes der Zugang zu dem electrischen Signale einer Reihe von Inductionsschlägen (des gewöhnlichen D u B o is'schen Inductionsapparates) eröffnet wurde, die das Metallfederchen des Signals ins Schwanken versetzten; die schnellen Schwankungen dieses Federchens bei relativer Langsamkeit der Cylinderrollung wurden nicht einzeln aufgeschrieben, sondern verschmelzen in einander und erscheinen daher in Gestalt eines weissen Streifens, dessen Breite der Amplitude der Schwankungen des zeichnenden Federchens, des electrischen Signales, entspricht. Mit dem Aufhören der Willensanstrengung behufs Acce-

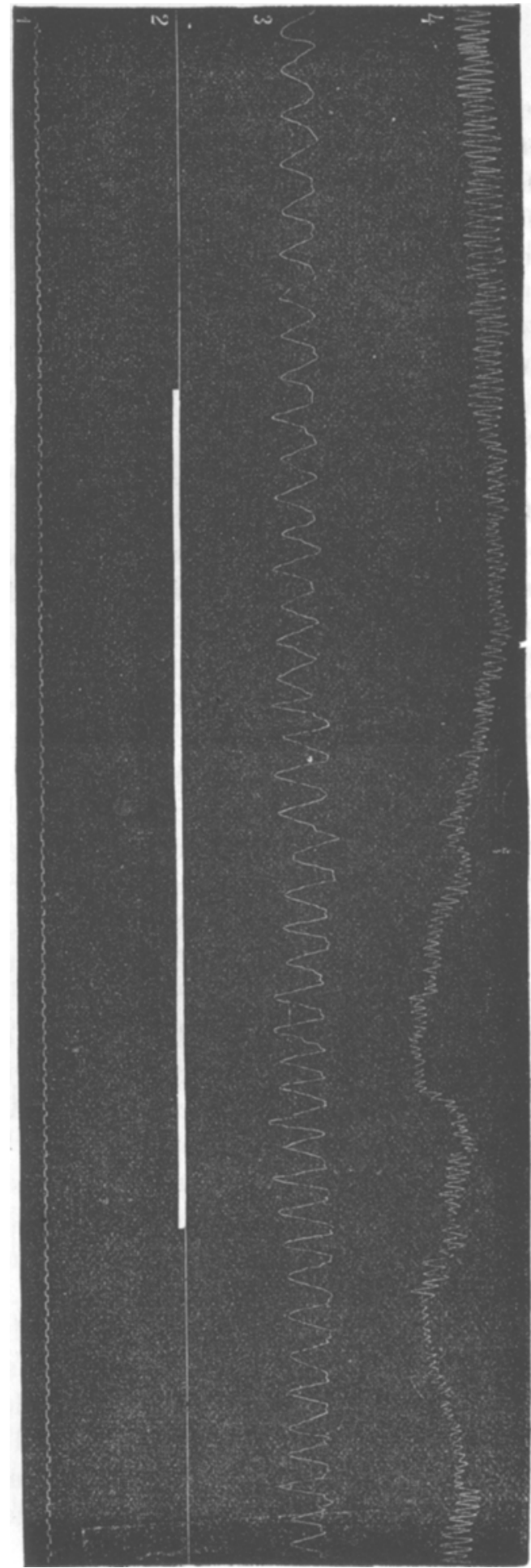

Fig. 1. 
Ueber die willkürliche Acceleration der Herzschläge beim Menschen. 117

leration des Herzschlags hörte der Untersuchte auf den Hebel anzudricken; durch das Herabsinken des anderen Hebelendes wurde der Strom aus dem electrischen Signal von Deprés augenblicklich ausgeschaltet und das zur Ruhe gekommene Federchen desselben fing von neuem an, eine weisse Linie zu zeichnen.

Die dritte Curve wurde mit dem Ma rey'schen Pneumographen, der auf dem Brustkorbe des untersuchten Individuums in der Höhe der Brustwarzen befestigt war, gezeichnet. Die Wellen dieser Curve geben genau Grösse und Rhythmus der Respirationsbewegungen wieder.

Die vierte Curve wurde mit Hülfe eines leicht modificirten Mosso-Frank'schen Plethysmographen, in den ein Fuss placirt war, niedergesehrieben und stellt Schwankungen zweierlei Art dar: erstens die des Pulses, zweitens die mehr ausgesprochenen Umfangsschwankungen der Organe. Die ganz deutlich verzeichneten Pulswellen erfordern keinerlei Commentarien; die Organverkleinerung drïckt sich in denselben durch Sinken der ganzen Curve aus, die Vergrösserung - durch Gesammtaufsteigen derselben.

Der in solcher Form angestellte Versuch macht es möglich, anschaulich zn bestimmen, wie gross die erhaltene Herzschlagsbeschleunigung sei, wie bald dieselbe dem erzeugenden Willensimpuls nachfolge, mit welcher Consequenz dieselbe sich entwickele und vergehe, und ob diese Beschleunigung von Veränderungen der Athembewegungen und Umfangsschwankungen der Extremitäten begleitet sei.

Figur 1 stellt nur ein Beispiel ans einer ganzen Reihe vor; ein Blick genügt, um zu ersehen, dass die Beschlennigung des Herzschlags mit den Veränderungen der Athembewegungen durchaus nicht verknüpft sein kann.

So haben wir vor Beginn der Beschleunigungsperiode im Laufe von zwanzig Secunden 32 Pulsationen auf 6 Athemzüge. Bei der Acceleration des Herzschlags haben wir 41 Pulsationen auf 7 Athemzïge im Lauf derselben Zeit, d. h. bei Berechnung auf die Minute erhalten wir 96 Pulsationen auf 18 Athemziige im ersten Fall, und im zweiten - 123 Pulsationen auf 21 Athemziige.

Es ist begreiflich, dass eine so auffallende Pulsbeschleunigung (um 27 Schläge in der Minute) in keinerlei Abhängigkeit von einer so unbedentenden Veränderung der Athembewegungen (Beschleunigung um 3 Athemzìge in der Minute) stehen könne. Unten an- 
zufiibrende Zahlen aus anderen, in meinem Besitz befindlichen Curven erhärten vollkommen diesen Sehluss. Beim Betrachten der Athemcurve bemerkt man leicht, dass der Character der Athembewegungen sich ein wenig ändert; dieselben werden ein wenig ungleich, die Höhe der Respirationswellen vergrössert sich ein wenig wider die Norm und erscheint dabei inconstant und es spitzen ausserdem die Gipfel dieser Wellen sich zu und weisen eben damit anf Verkürzung der Athempanse, eine Verkürzung, die mit der leichten Beschleunigung der Respirationsbewegungen während der Herzschlags-Beschleunigung vollkommen in Einklang steht. Uebrigens stellt diese Ligenthümlichkeit beim Betrachten einer grossen Anzahl Curven sich nicht als constant heraus und kann wegen der Geringítïgigkeit derselben in der analysirten Erscheinung der Herzschlags-Beschleunigung schwerlich irgend eine Bedeutung haben.

Zum Erweis unseres Gedankens erlauben wir uns, folgende aus anderen in unserm Besitz befindlichen Curven entnommene Zahlendata anzufiuhren.

Im Laufe von 20 Secunden:

Nr. des

Experimentes.

2

Zustand des zu Unter-
suchenden.

Ruhe

Willkïrliche Acceleration

1

Fortsetzen der willkürlichen

Acceleration . . .

6 Secunden nach Eintritt der

Ruhe

$\left\{\begin{array}{l}\text { Ruh } \\ \text { Wil }\end{array}\right.$

(Wiederum willkürliche Acceleration

3

Fortgesetzte willkürliche
Acceleration . . . $66^{1 / 2}$

6
Zahl der

Athemzüge. Herzschläge.
31

40

36

38

40

31.

Um an dem untersuchten Individuum von der Unabhängigkeit der Herzschlags-Beschleunigung von jeder beliebigen Anwendung der Athembewegungen noch mehr mich zu iberzengen, durchprobte ich den Einfluss der beziiglich Frequenz und Tiefe äussersten und 
Ueber die willkürliche Acceleration der Herzschläge beim Menschen. 119 auffälligsten Respirationsschwankungen auf den Rhythmus des Herzschlags, bei Benutzung derselben graphischen Methode.

Hier einige der erhaltenen Zahlen:

Im Laufe von 20 Secunden:

Zahl der Athemzüge.

Character derselben.

Zahl der Herzschläge.

22

$81 / 2$

9

8

0

0
Tief

Sehr oberflächlich . .

Mitteltief . . . .

$"$

$"$

Stillstand in der Inspira-

tion..... .

Stillstand in der Exspi-

ration
37

31

33

32

33

32

33.

Da auch diese ausserordentlich deutlichen Schwankungen der Athembewegungen im Rhythmus des Herzschlags nur schwach an unserem Subject sich abspiegelten, kann man sehwerlich daran zweifeln, dass die an ihm beobachtete, von seinem Willen abhängige Beschleunigung des Herzschlags in gar keiner Abhängigkeit von den dabei möglichen leichten Aenderungen der Athembewegungen stehe; diese letzteren Veränderungen erseheinen gleich. sam als eine beiläufige Complication, ähnlich der, die bei verstärkter willkürlicher Contraction beliebiger Muskelgruppen in der Sphäre der Athmung beobachtet wird.

An der plethysmographisehen Curve (Fig. 1, Curve 4) kann man leicht erkennen, dass der Act der Herzschlags-Beschleunigung einige Zeit nach seinem Beginn von deutlicher Umfangsverkleinerung der Extremität begleitet werde, eine Verkleinerung, die (bei der Bedingung vollkommener Ruhe der Extremität im Apparat) a priori entweder von Contraction der Blutgefässe der Extremität oder von einer verminderten, durch veränderte Herzthätigkeit bedingten Anfüllung der Blutgefässe abbängen könnte. Die Entscheidung, welche dieser Voraussetznngen richtig sei, war auf dem Wege der Messung des arteriellen Blutdrucks in der Extremität sowohl zur Zeit der Herzschlags-Beschleunigungsperiode, als anch vor nnd nach derselben möglich. Dazu benutzte ich, bei verbindlichster Mitwirkung der Drin. Schumowa das Sphygmomanometer von Basch, womit die Schwankungen des arteriellen Druckes in der Radialarterie bestimmt wurden. 
Es wurden folgende Resultate erhalten:

Experiment Nr. 1. In liegender Körperlage und bei vollkommener Ruhe des untersuchten Individuums schwankte der Blutdruck im Laufe von 3 Minaten zwisehen 105 und $110 \mathrm{~mm}$ der Quecksilbersäule; der Puls betrug während der ganzen Zeit 76 Schläge in der Minute.

Zur Zeit der Beschleunigungsperiode, welche den Puls von 76 auf 110 in der Minute erhöhte, erhob der Blutdruck sich allmählich von $110 \mathrm{~mm}$ auf $112,115,118,120 \mathrm{~mm}$, und nach Aufhören des auf die Acceleration aufgewandten Willensimpulses, als der Puls anfing zur Norm zuriickzukehren, erhielt der Blutdruck dennoch in den hohen Zahlen sich fort, und nachdem der Puls zur normalen Frequenz, d. h. zu 76 Seblïgen in der Minute schon gesunken war, sehwankte der Blutdruck gleichwohl zwischen 118 und 120 Schlägen noch fort. Erst 5 Minuten nach geschwundener Herzschlags-Beschleunigung kehrte auch der Blutdruck zur normalen Höhe, d. h. zu 105-108 mm der Quecksilbersänle zurïck.

Experiment Nr. 2. In diesem Experiment wurden Puls und Blutdruck für Zeiträume von je 15 Secunden bestimmt:

\begin{tabular}{|c|c|c|c|c|c|}
\hline \multicolumn{2}{|c|}{ Zustand des Cntersuchten. } & \multicolumn{2}{|c|}{ Zeit. } & Puls. & Druek. \\
\hline & Ruhe. & & Sec. & 20 & $110-112 \mathrm{~mm}$ \\
\hline & $"$ & 15 &, & 21 & $110-112$, \\
\hline & $"$ & 15 & $\eta$ & 20 & $110-112$ \\
\hline & hleunigung. & 15 & , & 21 & $118-120$ \\
\hline & , & 15 & $"$ & 22 & $120-125-128 \mathrm{~mm}$ \\
\hline & $"$ & 15 & $n$ & 25 & $130-132 \mathrm{~mm}$ \\
\hline &, & 15 & , & 26 & $130 \mathrm{~mm}$ \\
\hline & & 15 & $\eta$ & 24 & 132 \\
\hline & $"$ & 15 & , & 23 & $132 "$ \\
\hline : & 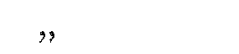 & 15 & $\eta$ & 22 & $125-122 \mathrm{~mm}$ \\
\hline E: & , & 15 & 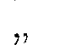 & 22 & $128 \mathrm{~mm}$ \\
\hline 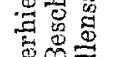 & 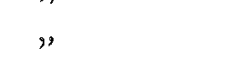 & 15 & 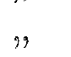 & 23 & $122-120 \mathrm{~mm}$ \\
\hline 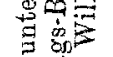 & $"$ & 15 & $\therefore$ & 21 & $132 \mathrm{~mm}$ \\
\hline & $"$ & 15 & , & 24 & $130-128 \mathrm{~mm}$ \\
\hline & $"$ & 15 & , & 24 & \\
\hline & , & 15 & , & 22 & $120-125 \mathrm{~mm}$ \\
\hline 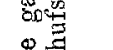 & , & 15 & , & 19 & $128 \mathrm{~mm}$ \\
\hline ब & $n$ & 15 & $\eta$ & 20 & $128-125 \mathrm{~mm}$. \\
\hline
\end{tabular}


Ueber die willkürliche Acceleration der Herzschläge beim Menschen. 121

Gegen das Ende ermüdete der Untersuchte und war nicht mehr im Stande, bei der allergrössten Energie irgend welche Herzschlags-Acceleration herrorzurufen.

Die Pulsfrequenz wurde, wie es ersichtlich ist, gegen Ende der Accelerationsperiode sogar geringer als die normale Frequenz während der Ruhe. Die ganze Dauer der auf die HerzschlagsBeschleunigung verwendeten Willensenergie betrug 12 Minuten; davon benutzte ich im Ganzen nur 3,75 Minuten zur Messung des Pulses und Blutdrucks.

55 Minuten nach Eintritt völliger Ruhe zeigte der Untersuchte in den nachstehenden, einander unmittélbar nachfolgenden Zeitperioden von je 15 Secunden:

$\begin{array}{cl}\text { Puls. } & \text { Blutdruck. } \\ 20 & 120-122 \\ 19 & 118-120 \\ 19 & 125 \\ 18 & 125 .\end{array}$

Experiment Nr. 3. Wurde nach einer halbstündigen Erholung nach den zwei ersten unternommen. Der zu Untersuchende fühlte sichtlich sich ermiidet und brachte desshalb nicht mehr eine so energische Beschleunigung hervor wie in den zwei ersten Versuchen. Aber dies Experiment gewährt den Vorzug, dass Puls und Blutdruck nach je 15 Secunden ununterbrochen die ganze Zeit hindurch verzeichnet wurden und es tritt darin die Unabhängigkeit der Herzschlagsbeschleunigung von der Höhe des Blutdrueks mit besonderer Schärfe hervor.

Zustand des Untersuchten. Zeit. Puls. Druck.

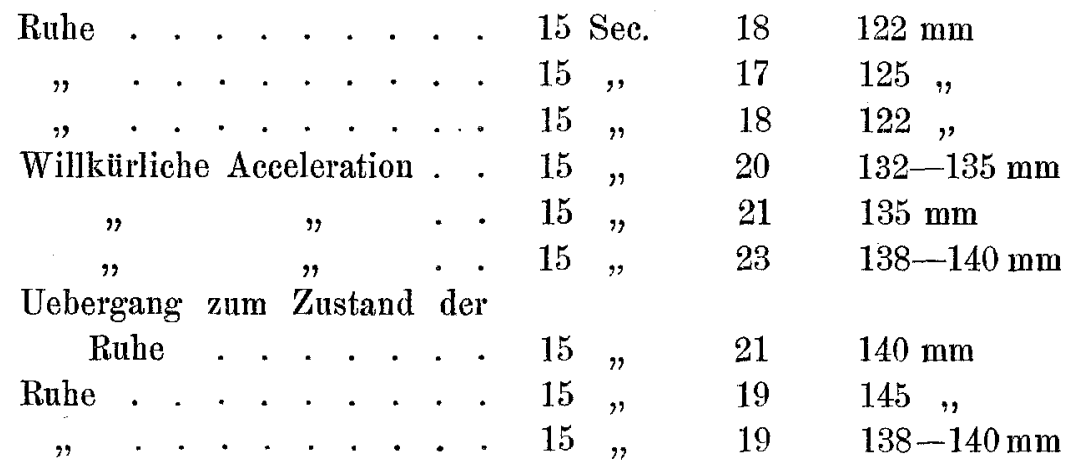


Zustand des Untersuchten. Zeit. Puls. Druck.

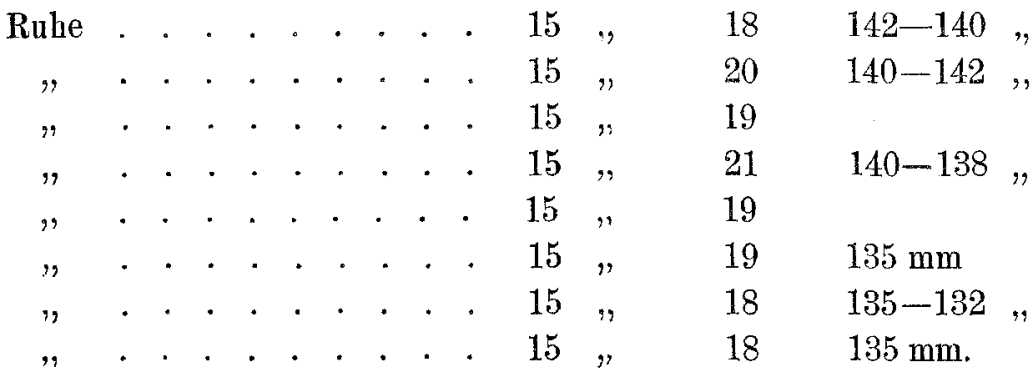

Alle drei hier vorgeführten Experimente zeigen übereinstimmend, dass mit Eintritt der Herzsehlags-Acceleration mehr oder weniger bald auch Blutdruckssteigerung sich einstelle, die auf längere Zeit die Herzschlags-Acceleration selbst überdauert; so konnte der Pulsrhythmus schon vollkommen zur Norm zurïckkehren, während der Blutdruck auf relativ hohen Zahlen sich forterhielt.

Diese Folgerung wird besonders anschanlich, wenn wir graphisch die Data auch nur des letzten Experiments darstellen.

Aus der graphischen Darstellung (Fig. 2) erhellt es, dass mit Beginn der willkürlichen Herzschlags-Acceleration auch der Blutdruck anfange sich stufenweise zu steigern und erst dann sein Maximum erreiche, wenn der Untersuchte die auf HerzschlagsBeschleunigung gerichtete Energie des Willens aufgehoben hat und wenn der Puls schon anfängt, eine deutliche Verlangsamung zu zeigen; diese Blutdruckserhöhung währt längere Zeit, zum Sinken eine geringe Neigung äussernd, und gegen Ende des Versuches, wo der Puls schon vollkommen zur Norm zurückgekehrt ist (im Zustande der Ruhe), hält der Blutdruck sich dennoch um $13 \mathrm{~mm}$ iiber seiner normalen Höhe. In dieser Beziehung coincidiren die Resultate der Blutdrucks-Messung mit den Andeutungen der plethismographischen Curve (Fig. 1, Curve 4), wo wir bemerkt hatten, dass die willkürliche Herzschlags-Beschleunigung von einer Umfangsverminderung der Extremität begleitet sei, welche Umfangsabnahme gleichfalls die Periode der Herzschlags-Beschleunigung überdauerte.

Die Aufzeichnungen des Sphygmomanometers, die eine der plethysmographischen Curve annähernd parallele Curve darstellen, 


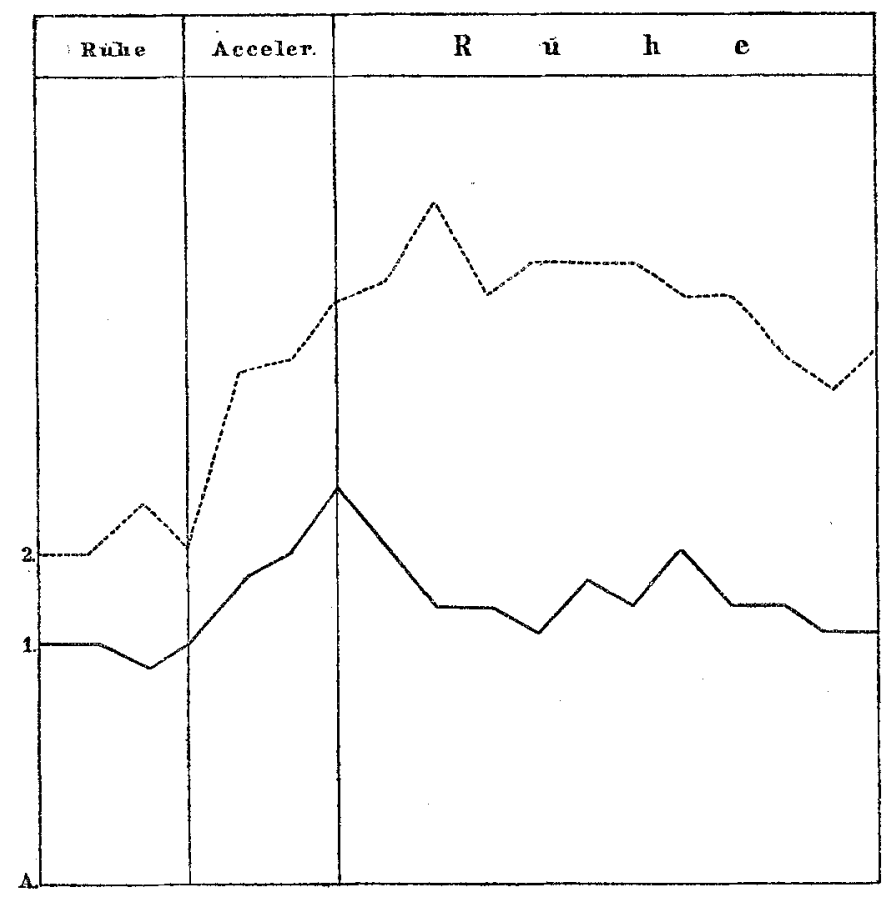

Fig. 2.

1. Die Curve der Puisschwankungen.

2. Die Curve der Schwankungen des Blutdrucks.

geben zugleich den Schlüssel zur Erklärung der ersteren plethysmographisehen Curve.

Es ist evident, dass die vasomotorische Erscheinung der Blutgefüssverringerung der diewillkürlicheHerzschlags-Beschleunigungsperiode begleitende und überdauernde Umfangsabnahme der Extremität zu Grunde liegt, da der Seitendruck in den grösseren Arterienstämmen dieutlich hierbei steigt. Unsere obige Voraussetzung, dass in Folge verminderter Herzthätigkeit die Umfangsabnahme der Extremität in der Beschleunigungsphase von ungentigendem Zufluss arteriellen Blutes abhängen könnte, verliert allen Boden schon in Folge obiger Experimente mit dem Blutdruck, da wir sonst nicht Erhöhung, sondern schnelles Sinken des Blutdrucks erhalten müssten, was in Wirklichkeit nicht eintritt. Wäre ausserdem die Umfangsverkleinerung der Extremität eine unmittelbare Folge der verminderten, das Blut ungenügend zuführenden Herzarbeit, so 
müsste mit dem unmittelbar nach der Beschleunigungsphase wiederkehrenden normalen Herzrhythmus die Wiederherstellung auch des Extremitätenumfanges eintreten. Allein diese Wiederkehr vollzieht sich in einer, dem stufenweise zur Norm sinkenden Blutdruck annähernd parallelen Reihenfolge.

Es ist also zweifellos, dass der Beschleunigungsact des Herzschlags von gleichzeitiger Erregung vasomotorischer Centra der Extremitäten begleitet wird, und dass der Effect dieser Erregung einige Zeit den willkürlichen Act der Herzschlags-Beschleunigung: iiberdauert. Da der innerhalb gewisser Grenzen erhöhte arterielle und somit auch intracordiale Blutdruck bekanntlich als Quelle der Herzschlagsbeschleunigung dienen kann, so erweist sich die Frage, ob nicht die Herzschlags-Beschleunigung auch in unserem Fall von dem erhöhten arteriellen Blutdruck abhängig sei, durchaus nicht als müssig. Mit anderen Worten, es könnte unser Fall in folgender Form präsentirt werden: Der Untersuchte versetzt die Arterien der Extremitäten in den Zustand der Contraction mittelst eines Willensaufwandes, erhöht den Blutdruck eben dadurch, letzterer aber bedingt seinerseits Beschleunigung des Herzschlags. Die HerzschlagsBeschleunigung erschien somit als eine nebensächliche, secundäre Erscheinung ohne directe Beziehung zur Willensthätigkeit.

Allein es ist leicht zu sehen, dass eine solche Vermuthung der Kritik nicht Stand halten kann. Aus der Vergleichung der Curve der Blutdrucksschwankungen mit der Pulscurve erhellt es, dass die Blutdruckserböhung der Herzschlags-Beschleunigung durchaus nicht vorausgeht, sondern nur dieselbe begleitet und um eine ziemlich beträchtliche Zeit überdauert.

Untersuchter konnte die auf Herzschlags-Beschleunigung gerichteten Anstrengungen bereits unterbrechen, aber der arterielle Druck fubr fort sich zu erheben und hielt sich auf relativ hohen Zahlen zu einer Zeit, wo der Pulsrhythmus schon zur Norm zurïckgegangen war. Es ist nun klar, dass es in unserem Fall keine Möglichkeit gebe, Erhöhung des Blutdrucks und Beschleunigung des Herzschlags wie Ursache und Wirkung zu verknitpfen.

Die vasomotorischen Erscheinungen des Untersuchten berührend, halte ich es nicht ohne Interesse, hinzuzufügen, dass die Hände während und nach der Phase der willkitrlichen HerzschlagsBeschleunigung sich kalt anfühlten, entsprechend der beschriebenen Gefässcontraction der Extremitäten; die mit einem Flächenthermo- 
Ueber die willkürliche Acceler tion der Herzschlägo beim Menschen. 125

meter gemessene Hauttemperatur fiel um $1-2^{\circ} \mathrm{C}$., während die Gesichts-, Stirn- und Wangenhant um $0,5^{0}$ C. u. m. sich erwärmte. Hier begegnen wir derselben Reihe vasomotorischer Erscheinungen, die jede heftige Nervenerregung begleiten, nämlich einer Verengung der peripheren Gefässe nebst Congestion zum Kopf.

Gleichfalls an dieser Stelle werde eine Erscheinung beiläufig angedeutet, die auf eine hohe Erregbarkeit des rasomotorischen Systems des Untersuchten hinweist.

In dem Zimmer, in dem die Blotdrucksuntersuchungen vorgenommen wurden, befanden sich einige Aerzte, die dann und wann mit Fragen halbla ut $z u$ einander sich wandten. Nichtsdestoweniger stieg der Blutdruck in der A. radial. um $5-15 \mathrm{~mm}$ der Quecksilbersäule und kebrte jedesmal nach eingetretener Stille von Neuem zur Norm zurtick. Diese Erscheinung erinnerte an die bekannten Mosso'schen plethysmographischen Untersuchungen, wo jede noch so wenig: heftige oder plötzliche Erregung des Nervensystems von einer Abnahme des Extremitätenumfanges, d. h. vou Contraction der peripheren Gefässe begleitet war. In unserm Fall wurde der vasomotorische Apparat unter dem Einfluss der schwächsten und kürzesten äusseren Gehörseindrïcke thätig.

Mit der hohen Erregbarkeit des vasomotorischen Systems ist die gleichfalls nicht geringere Erregbarkeit seines Herzens verknüpít. So schloss der Untersuchte die Lider zur Verlangsamung des Herzschlags (vor der Beschleunigungsphase desselben) und beseitigte jeden Lichtreiz. Dabei gelang es ihm dadurch, sein Ziel zu erreichen; oftmals schlug der Puls merklich gleichmässiger und manchesmal etwas langsamer. Oeffung der Angen ergab das Gegentheil. Ebenso beruhigend auf den Herzschlag wirkte die Concentrirung der Aufmerksamkeit auf irgend eine langweilige Abhandlung. $\mathrm{Zu}$ diesen Kunstgriffen nabm er nicht selten seine Zuflucht zur Beruhigung seines aus irgend welchem Grunde in Wallung versetzten Herzens.

Nachdem wir so alle wesentlichen Bedingungen, die auf unseren Fall von willkürlicher Beschleunigung der Herzaction Bezug hatten, erforscht haben, gelangen wir unvermeidlich zu der Folgerung; dass jene Acceleration kein Nebenresultat von Vorstellungen und Ideen (die rom Untersuchten hervorgerufen wurden), kein Resultat beliebiger Muskelbewegungen oder veränderter Athembewegungen oder veränderten Blutdrucks, sondern wahrscheinlich directe 
Folge der Willenseinwirkung auf die regulatorischen nervösen Herzcentra und Nervenbahnen des Herzens sei.

Es fragt sich nun, auf welche regulatorisehen Mechanismen der Wille in unserem Fall einwirkt. Die Acceleration des Herzschlags durch Willenseinfluss könnte entweder dadureh zu Stande kommen, dass das in der Medulla oblongata gelegene Herzhemmungscentrum durch die Willensimpulse unseres Individuums deprimirt, paralysirt, oder dass das in dem oberen Theil des Halstheils des Ruickenmarks gelegene Herzbeschleunigungscentrum erregt würde. Welche dieser beiden Erklärungen ist denn wahrscheinlicher? Zur Lösung dieser Frage mïssen wir erstens den Gang der Entwickelung der Acceleration unmittelbar nach Auftritt des beschleunigenden Willensimpulses und den Gang des Schwindens der Acceleration nach Aufhören desselben Willensimpulses verfolgen; zweitens müssen wir unsere Aufmerksamkeit auf die dabei auftretenden Veränderungen der Pulseurve, und drittens auf die Schwankungen des Extremitätenumfangs lenken.

In Betreff des ersten Satzes geniigt ein Blick auf die obige Pulseurve während der Acceleration des Herzschlags (Fig. 2), um zu ersehen, dass nach Einsatz des Willens die Pulsfrequenz nur allmählich steige und binnen $1 / 2-3 / 4$ Minuten, vom Anfange der Willensanstrengung gereclnet, das Maximum erreichen könne. Andererseits sinkt der Puls nach Aufheben dieses accelerirenden Willensimpulses gleichfalls allmählich und gelangt zur Norm binnen einigen Minuten, wobei beim Fallen noch einige secundäre Schwankungen nach der einen oder andern Seite sich zeigen. Dies allmähliche Auftreten und allmähliche Vergehen der Acceleration erinnert, dem Character nach, an die unmittelbare Rejzung des herzbeschleunigenden Mechanismus, d. h. der centrifugalen Fasern (nn. accelerantes) und deren Centra; es ist bekannt, dass auch in diesem Fall die Acceleration nicht mit einem Mal erhalten wird, sondern bis zu bestimmter Grenze allmählich anwächst, worauf die Beseitigung der Reizung ein ebenso allmähliches Schwinden der Acceleration verursacht, bis die Norm wieder erreicht wird. Ganz anders bei Lähmung der hemmenden Herznerven, d. i. bei Zerstörung des in der Medulla obl. gelegenen Herzhemmungscentrum oder bei Durchschneidung der NN. vagi. Hier wird der Puls fast sofort auffällig accelerirt und erreicht schnell das Maximum, und andererseits tritt bei Erregung des hemmenden Herzmechanismus, 
Ueber die willkürliche Acceleration der Herzschläge beim Menschen. 127 wenn die Erregung nur stark genug ist um überhaupt hervorzutreten, äusserst schnelle Verlangsamung des Herzschlags auf, d. h. es verschwindet die vorangehende Beschleunigung schnell. Beim Erwägen des Gesagten werden wir schwerlich irren, wenn wir sagen, dass in unserm Fall von willkürlicher Acceleration des Herzschlags der Effect durch Willenserregung der accelerirenden Herznerven erreicht werde.

Wir wenden uns zur Betrachtung des Pulses. Schon aus der plethysmographischen Curve der Pulswellen (Fig. 1, 4) kann man dentlich sehen, dass die Pulsbeschleunigung von allmählicher, aber auffälliger Abschwächung der Pulswellen begleitet werde, wobei in der Periode der bedentendsten Beschleunigung die Höhe derselben auf $1 / 3$ der normalen Höhe, die bei ruhigem Verhalten des Subjects beobachtet wird, und noch tiefer sinken kann. Da es wünschenswerth war, diese Pulswellenveränderungen genauer za verfolgen, registrirten wir dieselben sowohl mit dem Knoll'schen wie auch mit dem Marey'schen Sphygmographen. Da letzterer uns besser gelungene und glücklichere Curven lieferte, so bleiben wir bei Betrachtung letzterer.

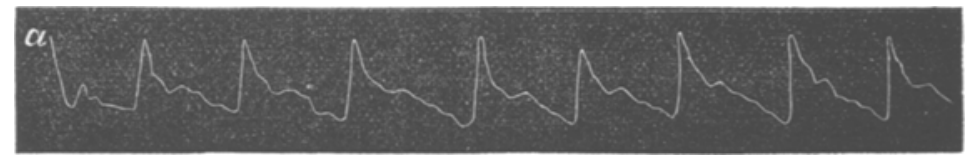

Fig. 3.

a - Puls im Zustande der Ruhe des Untersuchten; 76 in ider Minute.

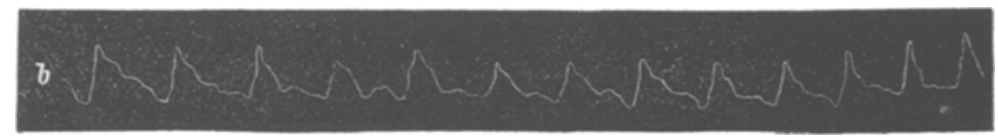

Fig. 4.

b - Puls inmitten der Acceleration; 105 in der Minute.

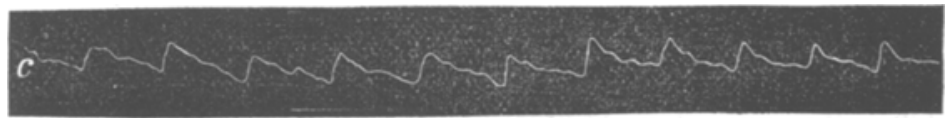

Hig. 5.

c - Puls zu Einde der Accelerationsperiode; der Untersuchte fühlt sich ermüdet. 


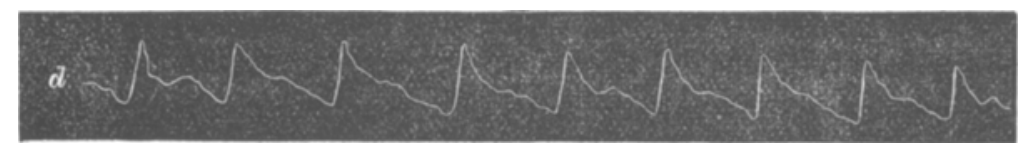

Fig. 6.

d - Puls, 5 Min. nach Aufhebung des accelerirenden Willensimpulses. Dex Untersuchte befindet sich im Zustand völliger Ruhe.

Beim Anblick angefübrter Pulscurven kann man klar erkennen, dass der accelerirende Willensimpuls Frequenz wie auch Character des Pulses ändere. Die systolische Erhebung oder Wellenhöhe vermindert sich allmählich nach Maassgabe der Beschleunigungsentwickelung der Herzschläge und sinkt annähernd auf $1 / 3$ der für den Ruhezustand normalen Höhe; ausserdem ist in der Beschleunigungsperiode die Steilheit der systolischen Erhebung gewöhnlich weniger auffallend ausgedrückt; diese beiden Umstände sind natürlich von einer weniger energischen Herzarbeit bedingt; wir sehen hier die anch bei künstlicher Erregung der beschleunigenden Herzmechanismen auftretende Erscheinung, wobei die einzelnen Pulsationen an Frequenz gewinnen, an Stärke verlieren. Gegen diese Erklärung könnte offenbar eingewendet werden, dass die Pulscurven-Veränderung Folge nicht der veränderten Herzarbeit, sondern der durch Contraction peripherer Blutgefüsse hervorgebrachten Blutdrucks-Veränderung sei, eine Contraction, die den HerzschlagsBeschleunigungsact bei unserem Individuum, wie wir sehen, begleitet. Eine solche Hypothese könnte darauf basirt sein, dass, je höher der mittlere Blutdruck steht, die anf den einzelnen Puls kommenden Druckzuwachsquoten und somit auch die Pulshöhe um so schwächer werden. Allein man kann aus folgenden drei Gründen hiermit nicht einverstanden sein: erstens waren die Zuwachsquoten des arteriellen Blutdrcks in der Beschleunigungsperiode so sehr unbedeutend (ca. $20-25 \mathrm{~mm}$ der Quecksilbersäule), dass die erhaltenen auffälligen Pulscharacteränderungen damit nicht erklärt werden dürfen; zweitens würde die weniger steile (mehr sanft geneigte) systolische Pulswellen-Erhebung in der Beschleunigungsperiode von diesem Gesichtspunkt aus unverständlieh bleiben; und drittens gehen die Characterveränderungen des Pulses, was am wichtigsten ist, mit den Schwankungen des Blutdrucks durchaus nicht Hand in Hand: nach Aufhören des accelerirenden Willensimpulses, wo der Puls zur Norm zurückgekehrt ist, hielt der 
Ueber die willkürliche Acceleration der Herzschläge beim Menschen. 129

Blutdruck in relativ hohen Zahlen sich fort. Diese Data genügen zu der Folgerung, dass die Characteränderung des Pulses (in der Beschleunigungsperiode) nicht von den Schwankungen des Blutdrucks, sondern von Aenderung der Herzarbeit abhänge.

In Betreff des absteigenden, dem diastolischen Sinken entsprechenden Pulscurven-Theils ist es ersichtlich, dass die Steilheit des Abfalles während der Herzschlags-Beschleunigung geringer wird, d. h. dass die Neigung beim Abfallen sanfter wird und dabei die secundären und tertiären Wellen, d. h. der Dikrotismus, Trikrotismus and an einzelnen Palswellen sogar Polykrotismus deutlicher hervortreten.

Diese beiden Erscheinungen im absteigenden Theil der Pulscurve erklären sich durch den verengten, gespannteren Zustand der peripheren Gefässe, der den Act der Herzschlagsbeschleunigung, wie wir sehen, begleitet. Und in der That müssen, in Folge der Gefässverengung, die Arterien unvermeidlich das Blut langsamer entleeren und somit eine schrägere Curve diastolischen Abfalls ergeben; andererseits muss der gespanntere Zustand der Arterienwände die Bildang secundärer Vibrationswellen und somit die Erscheinungen der Polykrotie begünstigen. So sprechen auch die Characteränderungen des Pulses (in der Beschleunigungsperiode des Herzschlags) dafür, dass unser Subject nicht durch Depression des hemmenden Herzmechanismus, sondern durch Erregang mittelst Willenseinflusses des accelerirenden Herzmechanismus das Ziel erreiche.

Für dieselbe Thesis spricht noch folgender indirecte Beweis: Bekanntlich ähnelt der durch Erregung des accelerirenden Herzcentrums hervorgerufene veränderte Herzschlag seinem Character nach dem durch Erwärmen desselben Organs erhaltenen auf's Aeusserste. Somit war es für mich höchst interessant, die beim Aufenthalt des Menschen in einer heissen russischen Badestube auftretenden Pulsveränderungen mit denen, die unser Subject durch Willenseinfluss hervorrief, zu vergleichen, da in derselben die Körpertemperatur eines jeden Menschen am $0,5-2{ }^{\circ} \mathrm{C}$. a. m. sich erhöht und somit das Herz die Wirkung erhöhter Temperatur erfährt. In der von Kostjürin über die physiologische Wirkung der russischen Bäder auf den menschlichen Organismus ausgeführten Arbeit ${ }^{1}$ ) finden sich vollkommen befriedigende Hinweise auf die

1) 1883, Juni, p. 32. - Meschdunarodnaja Klinika (russisch). 
Veränderungen von Frequenz wie Character des Pulses unter dem Einfluss einer (im Mittel auf $57^{\circ} \mathrm{C}$.) erhöhten Badestubentemperatur. Der Vergleich der von ihm erbaltenen sphygmographischen Curven mit den unsrigen, in der Periode der Herzschlagsbeschleunigung bei unserem Subject eingeschriebenen, ergiebt fast völlige Identität. Gleich den Pulsveränderungen unseres Individuums beim Uebergang aus Ruhe in willkürliche Acceleration des Pulses sind die Pulsalterationen beim Uebergang aus der gewöhnlichen Temperatur des Zimmers in die hohe Temperatur der Badestube; neben auffälliger Acceleration des Pulses, verändert sich auch der Character der Pulswellen: es verringert sich merklich die systolische Erhebung und diastolische Steilheit des Abfalls und es vermehrt sich Dikrotismus und diastolisches Abfallen bedentend. Kurz, es wird das von uns an unserem Individuum in der Periode der durch Willenseinfluss beschleunigten Herzaction erhaltene Resultat fast buchstäblich wiedergefunden. Was in unserem Fall blosse Willensenergie, vermag bei anderen Menschen die auf den Organismus (und ergo anch auf's Herz) wirkende Wärme zu erzeugen. Somit spricht die fast völlige Identität der oben verzeichneten, beim Erwärmen des Herzens in der Thätigkeit dieses Organes eintretenden Veränderungen, mit den bei directer Reizung der accelerirenden Herznerven an Thieren gewonnenen Resultaten, sowie andererseits die fast gleichartigen Pulsveränderungen sowohl bei durch Willenseinfluss hervorgerufener Acceleration, wie überhaupt bei gesunden Menschen, nachdem dieselben der Einwirkung einer hohen Badestubentemperatur ausgesetzt worden sind, zu Gunsten der Meinung, dass der Untersuchte durch seine Willensenergie die accelerirenden Herzmechanismen errege und dadurch den Herzschlag beschlennige.

$\mathrm{Zu}$ derselben Folgerung fiuhrt die obige Umfangsschwankung der Extremitäten unseres Subjects. Entsprechend der plethismographischen Curve haben wir gesehen, dass der Uebergang zur willkürlichen Pulsaceeleration von einer Abnahme des Extremitätenumfangs begleitet wird. Dieses Factum benimmt sofort jede Erklärungsmöglichkeit der Beschleunigung des Herzschlags in unserem Fall durch eine willkürliche Depression des hemmenden Herzmechanismus, da gerade der umgekehrte Effect, d. h. auffällige Umfangsvergrösserung infolge verstärkten Blutzuflusses in die Arterien nebst starker übereinstimmender Blutdruckserhöhung hierbei unvermeidlich wäre. Es zeigte sich aber in Wirklichkeit auffällige 
Abnahme des Extremitätenumfangs neost nur geringerer Blutdruckserhöhung.

Auf Grund obiger Thatsachen und Erfahrungen halte ich es für völlig gerechtfertigt, zuzulassen, dass Salo mé die merkwürdig seltene Gabe besitze, den accelerirenden Herzmechanismus willkürlich zu erregen. Es muss natürlich eine besondere Organisation des Nervensystems einer derartigen Fähigkeit za Grunde liegen. Die einfachste Erklärung dieses von uns angeführten Falles wäre, die Existens direeter Nervenverbindungen im Centralnervensystem unseres Subjects zwischen den höchsten Willenscentren der Hemisphärenrinde und den (in den oberen Theilen des Halsrïckenmarks gelegenen) accelerirenden Herzcentren zuzulassen. Es könnte überhaupt im menschlichen Nervensystem eine ähnliche intercentrale Verknüpfung existiren, nur wäre dieselbe in der ungeheuren Ueberzahl der Fälle für Willensimpulse nicht besonders durchgängig und infolge unbekannter ätiologischer Momente in unserm Fall der Willenserregung unterworfen.

Untersuchter beherrscht das Herz eig enthü ml icher We is e nur in einer Richtung, d. b. seitens der Acceleration, und vermag willkürlich auch nicht im Geringsten den Herzschlag zu hemmen.

Wir gehen nunmehr an die Beschreibung einiger, die willkürkürliche Beschleunigung des Herzschlags bei unserem Subject beeinflussender Momente.

Am leichtesten und mit bedeutendstem Erfolg wurde die Pulsbeschleunigung in der Regel des Morgens nach einer ruhig verbrachten Nacht, wo unser Subject sich vollkommen munter und ruhig fühlt und vor Aufnahme von Kaffee oder Thee oder sonstiger erhitzender Getränke, hervorgerufen.

Es ist bemerkenswerth, dass alle, so oder anders Ermidung oder Erschöpfung des Nervensystems überhaupt und des Herzens insbesondere bewirkenden Momente, wie verstärkte Muskelbewegung, angespannte Gehirnarbeit, erhitzende Getränke, sexuelle Excesse, vorangegangener fortgesetzter Aufenthalt in heisser Badestube, eine schlaflose Nacht, vermehrtes Tabakranchen, schwächten eigenthümlicher Weise auffällig die Fähigkeit unseres Subjects, den Herzschlag zu acceleriren. Unter diesen ungünstigen bier anfgezühlten Umständen kostete es ihm einen colossalen Willensaufwand, um den Puls auch nur um 10-18 Schläge in der Minute zu beschleunigen. In demselben ungünstigen Sinn wirkten auch un- 
mittelbar sich folgende Acte der Acceleration, von nur kurzen Pausen unterbrochen; mit jedem folgenden Act wurde die Acceleration geringer und war mit immer grösserer Anstrengung verbunden. Zuletzt war Untersuchter so ermüdet, dass er uns erklärte, vollkommen unvermögend geworden zu sein, irgend welche weitere Acceleration hervorbringen zu können.

Unter den die Fähigkeit unseres Subjects der willentlichen Acceleration des Herzschlags beeinflussenden Agentien verzeichnen wir noch zwei: Arsenik und Stickstoffoxydul.

Die Informationen über das erste Agens erhielt ich von Sa$10 \mathrm{mé}$ selbst, der bei Einnahme von Solutio arsenical. Fowleri in kleinen Dosen und einige Tage hindurch bemerkt hatte, dass dabei viel leichter die Acceleration des Herzschlags gelinge und dass dabei selbige viel auffälliger als an den Tagen, an welchen die Arzenei nicht eingenommen wurde, auftritt. Diese Wirkung zeigte sich so constant und sicher, dass in Fällen vorangehender Vorbereitung zur Demonstration seiner Fähigkeit der Herzschlagsacceleration, er zur Verstärkung des Effects bisweilen geringe Dosen Arsenik einnahm. Ich übernehme es nicht za erklären, wie Arsenik den Eintritt der willkürlichen Aceeleration der Herzschläge begünstigt, ob infolge erhöhter Erregbarkeit des accelerirenden Herzmechanismus oder durch Erhöhung des allgemeinen Wohlbefindens und vermehrter Energie des Organismus. So oder anders, aber in Folge dieser Beobachtung wäre es doch wünschenswerth, die Wirkung des Arseniks auf die Functionirung des accelerirenden Herzmechanismus zu untersuchen.

Die Erfahrungen über den Einfluss des Stickstoffoxyduls sind verbindlichst vom gewesenen Ordinator der Prof. Botkin'schen Klinik, Dr. Klikowitseh mir übermittelt worden. Fr unterwarf Salomé Inhalationen eines Gasgemenges von 4 Volumina Stickstoffoxydul und 1 Volumen Sauerstoff, d. h. eines solchen Gemenges, das bei völliger Garantie der Erhaltung der zur Respiration unentbehrlichen O-Menge und ohne das Bewusstsein zu trüben, dem Untersuchten die Möglichkeit gewährte, den Herzschlag zn beschleunigen. Es wurde vor der Inhalation die Zahl der Pulsschläge in der Minute gezählt und darauf die Acceleration der Schläge in derselben Zeiteinheit unter Einwirkung des Willensimpulses bestimmt. Unter gewöhnlichen Umständen konnte unser Subject den Puls um 20-30 Schläge in der Minute beschleunigen. 
Da es aus der Arbeit von Goldstein ${ }^{1}$ ) bekannt ist, dass Inhalationen von $\mathrm{N}_{2} \mathrm{O}$ dureh Abschwächung der herzhemmenden Vagusfunction per se den Herzschlag leicht acceleriren, so bestimmte Klikowitsch zuvor den Grad dieser Acceleration unter dem Einfluss von 6-10 Inhalationen obigen Gasgemenges. Bei einfacber Inhalation ohne jeden accelerirenden Willensaufwand wurde der Puls nur um 6-- 8 Schläge in der Minute frequenter. Nach einer Erholung von 15 Minuten wurden von Neuem 6-10 Inhalationen vom Untersuchten ausgeführt und nach Klikowitseh's Aufforderung Acceleration des Herzschlags angestrebt; allein die Versuche blieben ganz erfolglos, der Puls blieb vollkommen unverändert und diese Machtlosigkeit des Untersuchten dauerte trotz völlig bewahrten Bewusstseins, so lange, bis nach unterbrochener Inhalation der Einfluss des Luftgases völlig gewichen war; dann genügte derselbe Willensimpuls, um die Herzaction um 24-30 Schläge in der Minute wie zuvor' zu beschleunigen. Untersuchter definirte jene Machtlosigkeit nach Inhalationen von Sticksoffoxydul durch Vergleich derselben mit der vergeblichen Anstrengung, einem gelähmten Glied motorischen Willensimpuls zu ertheilen. In dieser Form wurde das Experiment 4 mal wiederholt and immer dasselbe Resultat erbalten; stets wurde durch Stickstoffoxydul das Vermögen, den Herzschlag willkürlich zu beschleunigen, vernichtet.

Zuletzt erscheint es, zur grösseren Vollständigkeit des Umrisses, nothwendig, die Resultate der durch Prof. Botkin an Salomé vorgenommenen klinischen Untersuchung anzuführen. Um des grossen Interesses willen wird die Beschreibung ${ }^{2}$ ) dieser Analyse des geschickten Klinikers wörtlich angeführt: "Untersuchter ist von hohem Wuchs, mittlerer Constitution und Ernährung, mit geringem Rest der Schilddrüse. Herzstoss diffus, nämlich zwischen der 3.--4., 4.-5. und am deutlichsten zwischen der 5. und 6. Rippe; die absolute Herzdämpfung beginnt unter der 3. Rippe und endet an der 6.; Querdurchmesser nach rechts fast bis zur l. mediana, nach links ganz bis zur l. mamillaris. In der linken Parasternalen eine leichte Dämpfung bemerkbar, die fast

1) Dies Archiv 1878, 17. Bd, 7. u. 8. Heft, S. 331.

2) "Klinische Wochenschrift", herausgeg. von Botkin. 1881. No. 10 (Russisch: Eschenedeinaja Klinitscheskaja Gazeta). 
unter der Clavicula beginnt, nach rechts zur l. mediana sich erstreckt und nach links bis auf 3 Fingerbreit vom Rand des Sternums entfernt ist. Die Leber beginnt von der 6. Rippe und überragt anscheinend den Rippenbogen. Milz percussorisch von der 8. Rippe, nicht palpirbar. In den Lungen vesiculäres Athmen; in der linken Partsternalen, in der Region der gefundenen Dämpfung etwas schwächeres und kürzeres Athmungsgeräusch als rechts. An der Herzspitze 2 Töne, an der Aorta und A. pulm. Accent auf dem zweiten Ton; in der linken Parasternalen unter der 3. Rippe systolisches Geräusch, bei Druck mit dem Stethoscop sich verstärkend. Beide Töne in den Carotiden. Puls $84 \mathrm{im}$ ersten Moment der Untersuchung, hob darauf sich rasch auf 96; ziemlich voll, weich und gleichmässig. Ueberhaupt abnorme Irritabilität des Herzens bemerkbar, denn es genügte eine einzige Untersuchung, um eine Pulsacceleration um 12-16 Schläge in der Minute hervorzurufen."

"Nachdem Salo mé sich einigermaassen beruhigt hatte, ergab die Wiederholung der objectiven Untersuchung nichts Abnormes." „Bei der accelerirenden Willensaction wird der Herzstoss schwächer und bleibt nur zwischen der 5. und 6. Rippe sichtbar, dabei zur 1. mamillaris näher; Längsdurchmesser des Herzens beginnt an der 3. und endet an der 6. Rippe; Querdurchmesser bat sich vergrössert, reicht ganz bis zur 1. mediana; das systolische Geräusch in der linken Parasternalen weniger dentlich, in der Region der Dämpfung aber erscheinen kleinblösige Rasselgeräusche, wobei die Dämpfung selbst deutlicher wird. Pulscharakter gleichfalls verändert in Frequenz und Kraft: von 94-96, beschleunigt anf 116, 118 und 120 Schläge; Puls weicher und schwächer geworden, leicht wegdrickbar; die einzelnen Pulswellen ungleich voll. Nach einer Erholung ergaben Percussion, Auscultation und Palpation das vor der willentlichen Acceleration gefundene Resultat."

Diese klinische Untersuchung bestätigt vollkommen, erstens die Resultate der vorangegangenen physiologischen Pulsuntersuchung, zweitens fübrt dieselbe durch Hinweis auf die Abschwächung des Herzstosses während der accelerirenden Willensaction zu derselben Folgerung, zu der auf einem andern Wege wir auch gelangt waren, dass nämlich die Accelerationsperiode des Herzschlags von Absehwächung der Herzarbeit begleitet wird, 
Ueber die willkürliche Acceleration der Herzschläge beim Menschen. 135

und drittens beweist dieselbe, was besonders wichtig ist, dass während der accelerirenden Willensthätigkeit solehe auffällige Veränderungen der Herzarbeit auftreten, die im Stande sind, sogar am Querdurchmesser dieses Organs sich zu zeigen. Letzterer Umstand veranlasste uns, die lange Reihe von Experimenten und Beobachtungen aufzugeben, die an H. S. auszufuhren wir im Sinne hatten, der die Untersuchung seines Herzens stets liebenswürdig proponirte und ihm zu rathen, weitere Uebungen in dieser Beziehung aufzugeben, zur Verhütung der allendlichen Entstehung eines Herzfehlers.

\section{Anhang.}

Dank der Liebenswürdigkeit einiger Aerzte, mit denen ich bekannt bin, ist es mir gelungen, noch einen anderen, viel bemerkenswertheren Fall von willkürlicher Acceleration der Herzschläge zu beobachten. Fin sehr nervöser und reizbar jungerMann vermochte beim ersten Probeversuch, den ich mit ihm anstellte, die Zahl seiner Pulsschläge nach Belieben von 85 Schlägen in der Minute auf 130 , d. h. auf 45 Schläge in der Minute zu erhöhen. Leider stellten sich bei ihm während dieser Probe seiner Fähigkeit den Puls zu beschleunigen, ein unwillkürliches Zittern des ganzen Körpers und sowohl frequentere wie auch energischere Athembewegungen ein. Dennoch war es nicht möglich, diese so beträchtliche Beschleunigung der Herzaction durch das Zittern oder durch die Aenderung des Characters der Athmung zu erklären, da die willkürliche Hervorrufung dieses Zitterns und der energischen und frequenten Athembewegungen allein nicht genügte, um eine Acceleration der Herzschläge zu Wege zu bringen; dazu war ein ganz specieller Willens. impuls des untersuchten Subjects unumgänglich nothwendig. Mir fiel noch ganz besonders Folgendes auf: ganz so wie bei dem ersten war auch bei diesem Individuum das Muskelsystem dem Willenseinflusse in viel weiteren Grenzen als bei der Mehrzahl der Menschen unterworfen; gleich dem H. Salomé war auch dieses Individuum im Stande die Ohrmuskeln ziemlich frei zu bewegen, ebenso auch die 3. Phalanx der Finger und verschiedene Muskelgruppen der Extremitäten und des Halses für sich allein zu contra- 
136 J. R. Tarchanoff: Ueb. d. willkürliche Acceleration d. Herzschläge etc.

hiren u. s. w. Demnach scheint mir bei diesem Subject ebenso die Abhängigkeit der Herzaction von gewissen beschleunigenden Willensimpulsen, eine specielle Function seiner allgemeinen neuromuskulären Organisation, welche sich gerade dadurch kennzeichnet, dass sie ganz besonders Willensimpulsen unterworfen ist, zu sein.

Da ich somit bemerkt hatte, dass bei beiden die Herzthätigkeit zu beschleunigen fähigen Subjecte, die ich untersucht hatte, gleichzeitig die ganz besonders auffallende Fähigkeit solche Muskelgruppen zu contrahiren, die bei der Mehrzahl der Menschen dem Willen nicht unterworfen sind, vorhanden war, fiel es mir ein, mich gerade dieses Kennzeichens beim weiteren Aufsuchen noch anderer, die Herzaction zu acceleriren fähiger Subjecte, zu bedienen. Die Thatsachen haben meine Erwartungen gewissermasser bestätigt. Ich begegnete einern Arzt S., der mit Leichtigkeit die Ohrmuscheln und die 3. Fingerphalanx bewegte und nervöser Constitution war; sogleich fragte ich ihn, ob er denn anch im Stande sei, seine Herzschläge willkürlich zu beschleunigen? Da er mir antwortete, dass er es noch nie versucht hatte, so bat ich ihn, sogleich seine Herzaction za acceleriren; schon beim ersten Versuch beschleunigte er dieselbe um mehr als 20 Schläge in der Minute. Nachdem er sich aber etwas eingeübt hatte, nngefähr während eines Monats, brachte er es so weit, dass er während eines meiner öffentlichen Vorträge in Gegenwart einer grossen Menschenmenge willkürlich die Zabl seiner Pulsschläge von 85 auf 160 Schläge in einer Minute erhöhte, d. h. somit fast verdoppelte.

Nachdem ich noch mehrere andere Personen, die die Fähigkeit besassen, ebenso mehr oder weniger auffallend die Muskeln, welche gewöhnlich dem Willenseinflusse nicht unterliegen, zu beherrschen, beobachtet hatte, fand ich noch 2 Menschen, - der eine ein junger russischer Dichter, der andere ein junger Professor K., - welche willkürlich ibre Herzthätigkeit auf 15-20 Schläge in der Minute zu beschleunigen vermochten. Bis jetzt aber ist es mir noch nicht gelnngen solche Menschen aufzufinden, die trotz einer ganz gewöhulichen netromuskulären Organisation dennoch im Stande würen, die Herzschläge willkürlich zu acceleriren. Aus diesem Grunde scheint es mir sehr wabrscheinlich, dass solche „Beschleuniger" der Herztbätigkeit sehr oft in der Zahl der Mitglieder der Theaterwelt, die ja gewöbnlich in einem höheren Maasse und mit einer unvergleichlich grösseren Geschicklichkeit und Kunst als 
Lente anderer Specialitäten ihre Muskelsysteme beherrschen, aufzufinden wären. Es wäre deswegen sehr wünscbenswerth, wenn Personen und ganz besonders Aerzte, welche freien Zutritt zur Theaterwelt besitzen, diesen Gedanken einer Prüfung unterziehen wïrden.

Aus diesen meinen persönlichen Beobachtungen habe ich die feste Ueberzeugung gewonnen, dass Fälle einer willkürlichen Acceleration der Herzthätigkeit viel öfter als man bis jetzt angenommen hat, vorkommen, und dass sie nur deshalb so unbemerkt geblieben sind und bleiben, weil es den solch' einer Beschleunigung fähigen Individuen, sowohl wie denen mit ihnen verkehrenden Menschen nicht eintälit, ihre Aufmerksamkeit darauf zu lenken.

Vom theoretischen Standpunkte erscheint jetzt die Thatsache der Abhängigkeit des Herzens von gewissen Willensimpulsen sehr naturgemäss, da in neuerer Zeit die mittels des Telephons an Muskeln und Nerven von Wedenski ansgeführten Untersuchungen bewiesen haben, dass im $\mathrm{N}$. Vagus auch rein motorische Fasern zum Herzen verlaufen.

\section{Wie sind die Erscheinungen zu verstehen, die nach Zerstörung des motorischen Rindenfeldes an Thieren auftreten?}

Von

\section{Dr. W. Bechterew,}

Docent an der Kaiserlich medicinischen Academie zu St. Petersburg, Ordinator der Klinik von Prof. Mierzejewsky.

Die Anschaung, dass in dem sogen. motorischen Rindenfeld der Grosshirnhemisphären echte Bewegungscentren enthalten seien, die von mir in einer kurzen vorlänfigen Mittheilung (Neurolog. Centralbl. 1883, No. 18) ausgesprochen und noch friber von vielen 